\title{
Implementation of stepdown therapy at the Health Sciences Centre, Winnipeg, Manitoba
}

\author{
LE NICOLLE MD FRCPC
}

$\mathrm{S}$ EPDOWN THERAPY IS WIDELY USED AT THE HEALTH Sciences Centre in Winnipeg. This approach to antimicrobial management, however, has been neither formalized nor systematically evaluated. Efficient transfer from intravenous to oral antimicrobial therapy has been the standard of care for acute pyelonephritis

Division of Infectious Diseases, Department of Medicine, Health Sciences Centre, University of Manitoba. Winnipeg. Manitoba

Correspondence: Dr LE Nicolle, MS-675D, Health Sciences Centre, 820 Sherbrook Street, Winnipeg, Manitoba R3A 1R9, telephone 204-787-4655, Fax 204-787-4699 and pediatric bone and joint infections for many years. Intense pressure to avoid or decrease duration of hospitalization has lead to widespread acceptance of the concept of stepdown therapy by most attending physicians and it is used for pneumonia, skin and soft tissue infections, adult bone and joint infections, and intraabdominal infections. The lack of a systematic program or evaluation means reliable data on extent and appropriateness of the practice is not available. General observations, however, suggest a pervasive appreciation of stepdown therapy. For instance, a request to identify appropriate oral therapy for patients receiving parenteral therapy is a frequent reason for infectious 
disease consultation by the surgical services. In the emergency room, patients who are otherwise stable would previously have been admitted for treatment of severe skin and soft tissue infections. Currently such patients are frequently asked to return to the emergency room for parenteral therapy two or three times daily for 24 to $48 \mathrm{~h}$, then are continued on oral therapy once there is evidence for improvement. Thus, there have been substantial changes in use of parenteral therapy in the past several years. While antimicrobial cost is one factor which has promoted this altered approach, pressures to decrease hospitalization and length of stay are likely more important contributors.

Some formal approaches to stepdown therapy have been attempted at our institution. The Health Sciences Centre has a restrictive antimicrobial formulary which requires infectious disease consultation or approval for virtually all expensive parenteral antimicrobials. Thus all patients receiving imipenem, ceftazidime, parenteral ciprofloxacin, ceftriaxone or cefotaxime and some antiviral agents are followed by the infectious diseases service. Antimicrobial therapy for these patients is changed to less costly alternatives, either parenteral or oral, as soon as clinically feasible. Different approaches to clinical practice by different infectious disease consultants, however, limits the consistency of this approach. In addition, the pharmacy distributes to all attending physicians and housestaff a booklet updating antimicrobial resistance and formulary practices at the Health Sciences Centre. One page of this book provides a list of oral therapy options for selected parenteral antimicrobials and estimates the cost differential per day for oral rather than parenteral regimens. The extent to which this information is used is not known.

It must be acknowledged, however, that stepdown therapy is only one component of an antimicrobial usage program. It should be integrated into the general institutional approach to antimicrobial therapy. For instance, at the diabetic foot clinic at the Health Sciences Centre we see many infected ulcers, and over $50 \%$ of these patients have osteomyelitis. This is one group where 'stepdown' therapy should have a major role. In fact, of these patients, $60 \%$ receive only oral antimicrobials. The remaining $40 \%$ of patients who require parenteral therapy are equally divided between those who are initiated on oral therapy and subsequently receive parenteral therapy, and those initiated on parenteral therapy who subsequently receive oral therapy. The more appropriate focus would seem to be a global antimicrobial approach to specific clinical presentations, rather than a pharmacological focus of changing parenteral to oral therapy.

Current approaches to decision making in health care use outcome assessments whenever feasible. Despite the apparent 'obvious' benefits of rapid transition from parenteral to oral therapy, clinical studies documenting the effectiveness of this approach and defining where it has greatest utility are, generally, lacking. Until such studies are available, we should maintain a cautious and critical approach to stepdown therapy and understand that neither the benefits nor limitations have been adequately defined. 


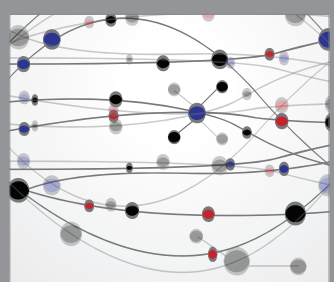

The Scientific World Journal
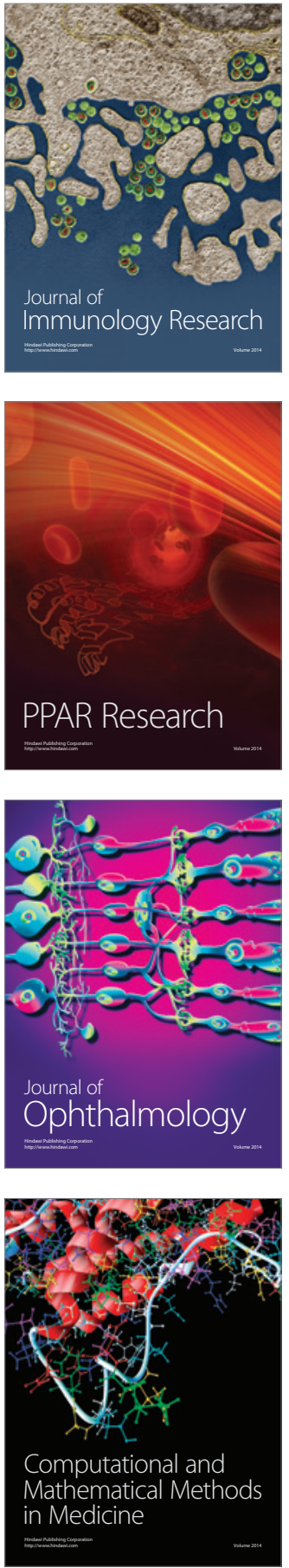

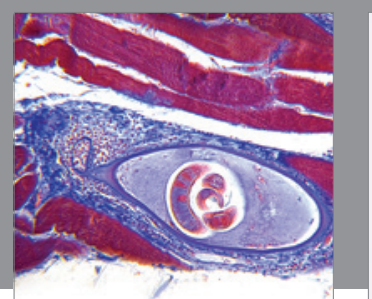

Gastroenterology Research and Practice

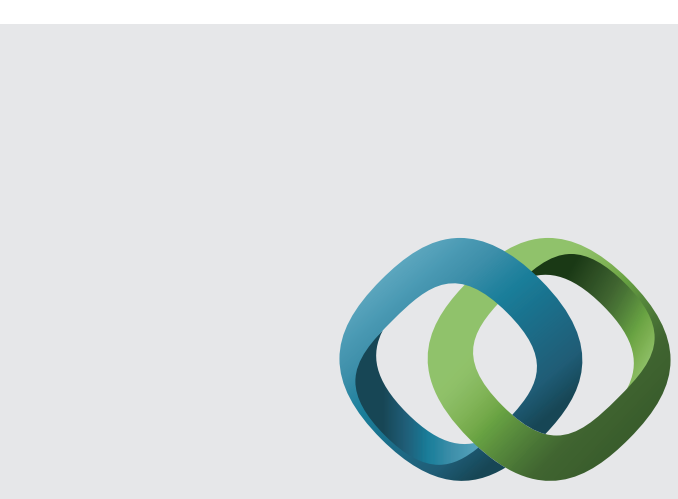

\section{Hindawi}

Submit your manuscripts at

http://www.hindawi.com
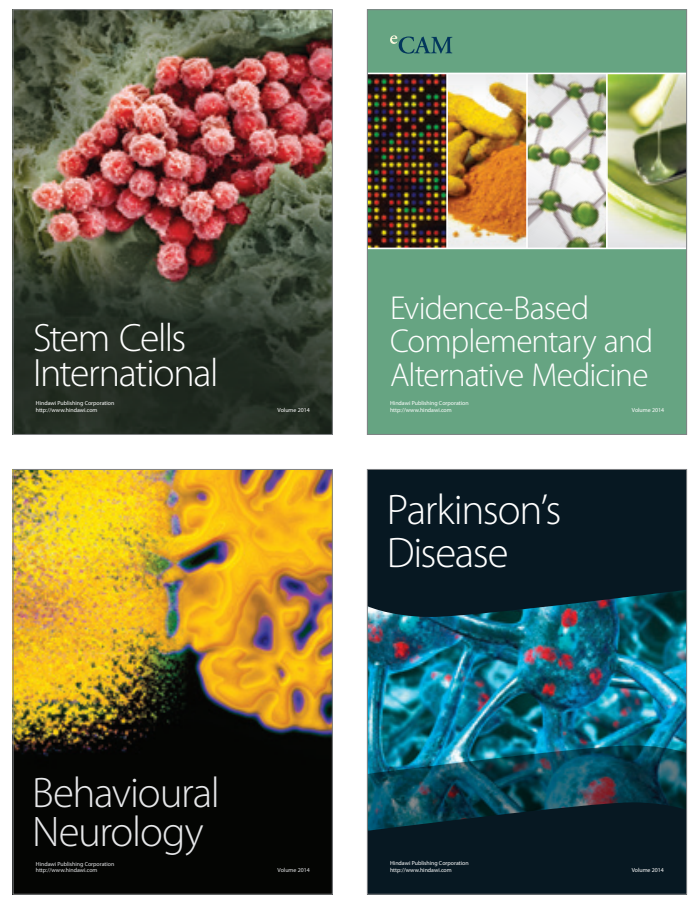
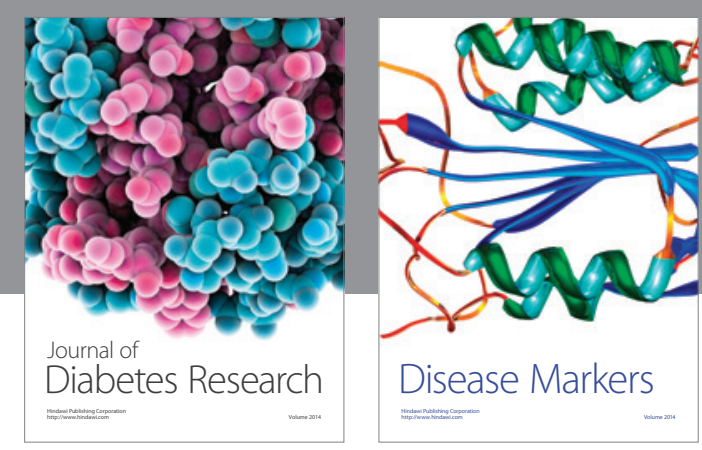

Disease Markers
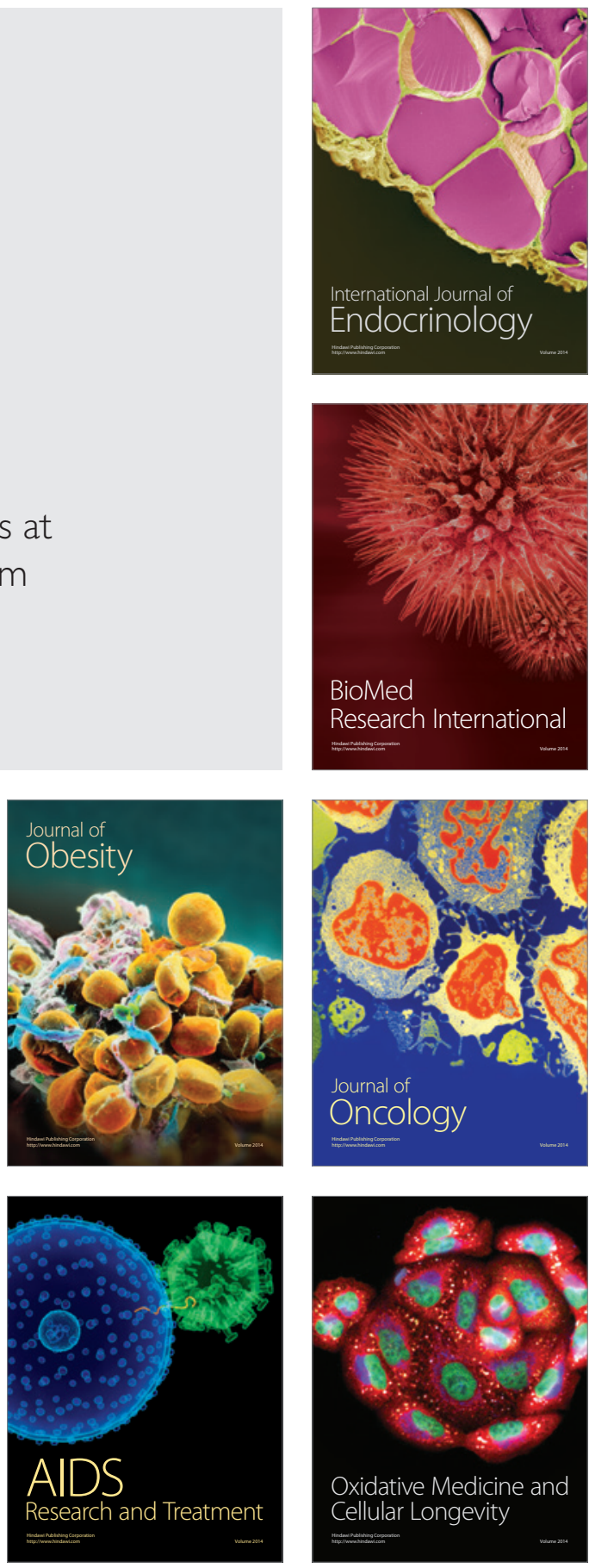